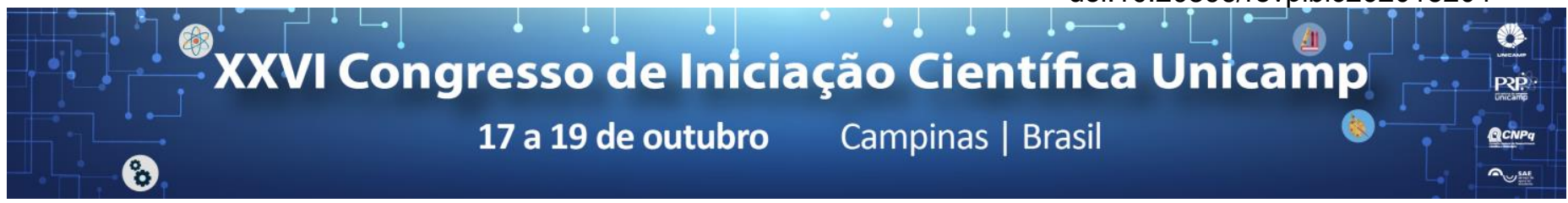

\title{
Analysis of the Runx3 expression during experiemental autoimmune encephalomyelitis
}

\author{
Beatriz S. Lopes*, Ana M. Marques, Alessandro S. Farias
}

\begin{abstract}
Multiple sclerosis (MS) is a chronic demyelinating disease of the central nervous system (CNS), which is attributed to a self-sustaining autoimmune mechanism. The majority of the knowledge regarding MS autoagressive mechanisms is resultant of studies performed in its experimental model (mice) the experimental autoimmune encephalomyelitis (EAE) . Although, the literature reports that the EAE is mediated by proinflammatory CD4+ T cells, isn't completely clear how these cells (known as helper cells) would be able of initiate the disease when adoptively transferred to healthy animals. We believe that the Runx3 gene presents a central role in the progression and regulation of the effector activity in CD4+ encephalitogenic lymphocytes.
\end{abstract}

\section{Key words:}

Runx3, autoimmunity, TCD4+ lymphocytes.

\section{Introduction}

At first EAE was considered as a Th1 mediated pathology. This was assured by data showing abundant IFN- $y$ in CNS lesions of mice with EAE and in patients with MS. Soon rapidly after its discovery, Th17 subset has been shown to perform an important role during EAE and other autoimmune pathologies. Apparently, Th1 and Th17 cells present a synergic role during EAE development. In addition, we found that some TCD4+ cells infiltrating the CNS were expressing the CD8 molecules.

In the thymus, the balance between ThPOK and Runx 3 is responsible for the destination of $T$ cell during the development. Our results showed that during the EAE evolution the relative Runx3 expression increases while the ThPOK expression remains constant. It is possible that different splice variants are regulating the processes in maturation of T CD4+ lymphocytes Thus, our objective to identify Runx3 pre-mRNA splicing variants in TCD4+ cells during the EAE evolution in comparison to single T CD4+ OR CD8+ positive cells at thymus.

\section{Results and Discussion}

Reporter animals with yellow fluorescent protein (YFP) coding sequence inserted at the Run $\times 3$ locus are being maintained. The insertion of the fragment was verified by the polymerase chain reaction (PCR) and confirmed by flow cytometry (image 1).

Image 1. Flow cytometry of HT Runx3 YFP mice spleen cells, with cells expressing YFP

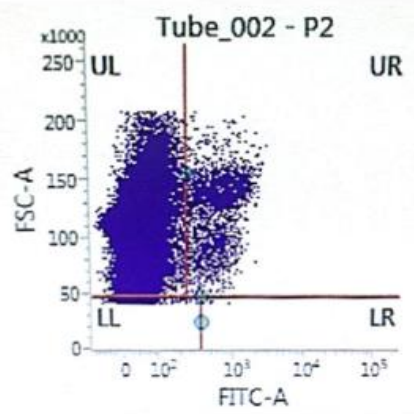

We initiated the Runx3 pre-mRNA splice identification using standard PCR. Specific primers were designed at the beginning and ending of the transcripts, for the identification of possible exons lost at this interval.

Although the PCR standardization has been one of the main obstacles found at this point of the project, we found stripes differing from the transcript size when testing the primers (image2).

Image 2. - Electrophoresis in agarose gel 1,5\% of the cDNA PCR with stripes of approximately 500pb and 900pb

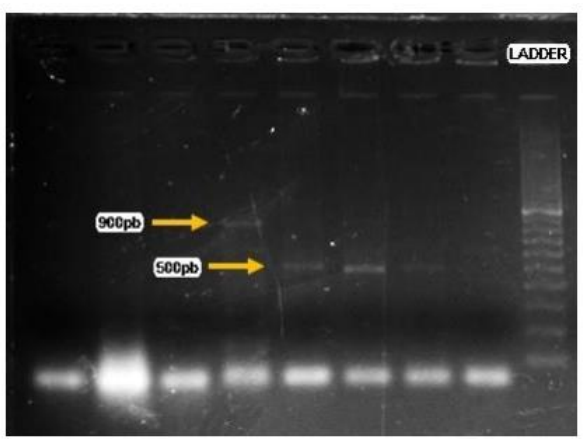

We believe that the smaller stripes than the transcript stripes indicate that there is a loss of exons, which could result in different Runx3 transcripts from those known so far. The next step it is use the SMARTer ${ }^{\circledR}$ RACE 5'/3' kit which can identify the different isoforms with greatest efficiency, even those less expressive ones.

\section{Conclusions}

We believe that the smaller stripes than the transcript stripes indicate that there is a loss of exons, which could result in different Runx3 transcripts then the ones that are know so far.

\section{Acknowledgement}

We would like to thank the CNPq/PIBIC program for the financial support and the members of the Neuroimunomodulation Lab of the University of Campinas.

Baxter, A. G. The origin and application of experimental autoimmune encephalomyelitis. Nature Reviews Immunology 7, 904-912 (2007).

Pierson, E., Simmons, S. B., Castelli, L. \& Goverman, J. M. Mechanisms regulating regional localization of inflammation during CNS autoimmunity. Immunological Reviews 248, 205-215 (2012). 